\title{
Screening, isolation and selection of cellulolytic fungi from oil palm empty fruit bunch fibre
}

\begin{abstract}
Screening and isolation of cellulolytic fungi was done using compost of oil palm empty fruit bunch conducted at a local factory located in Sri Ulu Langat, Dengkil, Selangor, Malaysia. This research was aimed to isolate of cellulolytic fungi with over production of cellulase components. Ten isolated fungi had shown the ability to degrade cellulose base on decolorization of CMC selective agar using Grams iodine as color indicator and cellulase production in shake flask fermentation. Aspergillus (R4) was selected as over producer of cellulase enzyme among ten isolated fungi. The Aspergillus (R4) with highest clearing zone on CMC agar $(50 \mathrm{~mm})$ and cellulolytic activity (CMCase 3.05, FPase 0.61 and b-glucosidase $1.75 \mathrm{U}$ mL-1) was identified as Aspergillus terrus. We identified Aspergillus terrues as highly cellulolytic in producing cellulase activity. Identification of over producers of cellulase enzymes can help industries exploits of this enzyme.
\end{abstract}

Keyword: Isolation; Celluloytic fungi; Cellulase; Oil palm empty fruit bunch; Fermentation 